Editorial

\title{
Single-Cell Profiling Explore the Immunologic Mechanisms of Tumor Relapse in Hepatocellular Carcinoma
}

\author{
Liyi Zhang ${ }^{1}$ \\ ${ }^{1}$ State Key Laboratory of Oncology, Collaborative Innovation Center \\ for Cancer Medicine, Sun Yat-sen University Cancer Center, \\ Guangzhou, Guangdong, People's Republic of China
}

Global Med Genet 2021;8:83-84.

Despite the worldwide decrease in liver cancer incidence and mortality over the past decade, it is still the fourth leading cause of cancer-related death. ${ }^{1}$ The burden of liver cancer is still serious, especially in China. ${ }^{2}$ In 2018, more than half of the liver cancer cases and deaths occurred in China. ${ }^{3}$ Hepatocellular carcinoma (HCC) is the most common subtype of liver cancer, accounting for $75 \%$ of all liver cancer cases. ${ }^{4}$ Surgical resection is the first choice for early-stage HCC, but more than $70 \%$ of HCC patients develop recurrent cancer within 5 years after surgery, ${ }^{5}$ which is the major cause of death in long-term evaluations. Among them, nearly $70 \%$ relapsed cases develop an early recurrence within 2 years of surgery. ${ }^{5}$ However, the precise molecular mechanisms of rapid recurrence remain unclear.

Tumor immune microenvironment (TIME) consists of various immune cells; education of tumor cells has become a hot topic in cancer research due to its association with initiation, progression, and recurrence. ${ }^{6}$ Therefore, profiling the immune contexture of HCC, which is determined by the composition, density, and functional orientation of tumor-infiltrating immune cells, could provide insights into immune escape mechanisms and helpful to design effective therapeutic strategies for recurrent HCC. Single-cell RNA sequencing (scRNA-seq) is the most powerful tool for tumor immune contexture profiling. ${ }^{7}$ With broad implications for both basic and clinical cancer research, scRNA-seq has already impacted our conceptual understanding of cancer progression in several cancer types. Although the immune contexture detected by scRNAseq in primary HCC has been reported, ${ }^{8}$ that in early-relapse HCC is still unknown.

In a study recently published in Cell, titled "Single-cell landscape of the ecosystem in early-relapse hepatocellular carcinoma," Sun et $\mathrm{al}^{9}$ examined 16,498 single-cell transcriptomes from 12 primary and 6 early-relapse HCC tumor tissues by scRNA-seq to define immune contexture at the single-cell resolution level. They found that early-relapse HCC had a distinctive immune contexture that is different from primary HCC. Compared with primary HCC, the density of regulatory T-cells (Tregs) was decreased and those of dendritic cells (DCs) and infiltrated $\mathrm{CD}^{+}{ }^{-}$-cells were increased in early-relapse HCC. Additionally, $\mathrm{CD}^{+}$T-cells in primary HCC displayed a classical exhausted state, but those in early-relapse HCC overexpressed CD161 and resided in an innate-like phenotype with low clonal expansion and cytotoxic state. These alterations were associated with a worse prognosis in HCC. The authors further explored the potential mechanisms of immune escape in early-relapse HCC. They found that tumor cells in early-relapse HCC could evade the host immune system's detection and destruction through suppressing DC-mediated antigen presentation and recruiting $\mathrm{CD} 161^{\text {high }} \mathrm{CD} 8^{+} \mathrm{T}$-cells.

The high incidence of early recurrence is the main reason for the poor clinical outcomes of HCC. Ignoring the differences between recurrent and primary tumors, recurrent HCCs are often treated based on the pathological characteristics and molecular classification of the primary HCCs. Although previous studies have reported that similar genomic alterations occurred in both primary and early-relapse $\mathrm{HCCs},{ }^{10}$ whether primary and early-relapse HCCs have differences in immune contexture is still unknown. The present study dissected and compared the TIME of primary and early-relapse HCCs by scRNA-seq. Unlike the findings in primary HCC reported in the previous studies (the primary HCC cells recruit Tregs to suppress the immune response $^{8}$ ), this study found that Tregs were excluded and $\mathrm{CD} 161^{\text {high }} \mathrm{CD}^{+}$T-cells were recruited to create an immunosuppressive microenvironment in early-relapse HCC. In CD161 ${ }^{\text {high }} \mathrm{CD}^{+} \mathrm{T}$-cells from recurrent tumor tissues, the expression levels of proliferation- and cytotoxicity-associated
Address for correspondence
Liyi Zhang, MD, PhD, State Key
Laboratory of Oncology,
Collaborative Innovation Center
for Cancer Medicine, Sun Yat-sen
University Cancer Center,
Guangzhou, Guangdong 510060 ,
People's Republic of China
(e-mail: zhangly1@sysucc.org.cn).
DOI https://doi.org/ 10.1055/s-0041-1729545. ISSN 2699-9404.

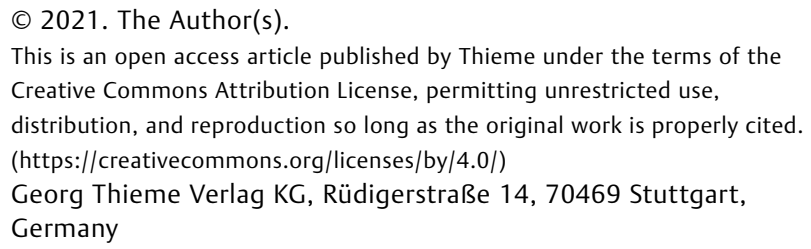


genes were significantly reduced and tissue residence-associated genes were increased as compared with those in $\mathrm{CD}^{+} \mathrm{T}$ cells from primary tumor tissues. CD161 is reported to be a surface marker for $\mathrm{CD}^{+} \mathrm{T}$-cells with innate-like and memory phenotype. ${ }^{11}$ In microbial infection, CD161 ${ }^{\text {high }} \mathrm{CD}^{+}{ }^{\mathrm{T}}$-cells contribute to the early control of microbial recall infections in both antigen-dependent and -independent manners. ${ }^{12}$ Therefore, the adaptive immune response of these cells is considered to be one of the important components of the immune response to the infectious diseases that have ever suffered. ${ }^{13}$ However, regardless of the obvious increase of $\mathrm{CD} 161^{\text {high }} \mathrm{CD}^{+} \mathrm{T}$-cell infiltration, HCC early recurrence can be achieved, which indicates that these T-cells cannot prevent the spread of HCC. The following observations of this study reveal why $\mathrm{CD} 161^{\text {high }}$ $\mathrm{CD}^{+}$T-cells with memory phenotypes cannot prevent HCC recurrence: (1) compared with $\mathrm{CD} 161^{-} \mathrm{CD} 8^{+} \mathrm{T}$-cells, CD161 ${ }^{\text {high }}$ $\mathrm{CD}^{+}$T-cells had a weaker antitumor ability, which was manifested as a decrease in the production of granzyme B and (2) T-cell receptor analysis indicated that CD161 ${ }^{\text {high }} \mathrm{CD} 8^{+}$ T-cells in early-relapse HCC reduced clonal expansion and shared most of T-cell receptor clone types with $\mathrm{CD}^{+}{ }^{+} \mathrm{T}$-cells in primary HCC. These findings indicated that $\mathrm{CD} 161^{\text {high }} \mathrm{CD} 8^{+}$ T-cells had the ability to target the clonal neoantigens in primary HCCs, but they could not be effectively activated in early-relapse HCCs.

DCs are critical antigen presenting cells. They play a key role in the regulation of the balance between $\mathrm{CD} 8^{+} \mathrm{T}$-cell immunity and tolerance to neoantigens. In the present study, enhanced effector functions of $\mathrm{CD}^{+} \mathrm{T}$-cells were not associated with the increased DC infiltration in early-relapse HCC and suggested that the antigen presentation functions of DCs were impaired in early-relapse HCCs. As we know, CD28 plays a central role in the regulation of T-cell activation and inhibition. ${ }^{14}$ Previous study found that programmed death-ligand 1 (PD-L1) has a stronger affinity for CD80 when compared with $\mathrm{CD} 28 .{ }^{15}$ As early-relapse HCC exhibited more PD-L1 ${ }^{+}$tumor cells than primary HCC, CD80 on a DC might preferentially bind to PD-L1 on early-relapse HCC cells rather than $\mathrm{CD} 28$ on a resting T-cell. Therefore, $\mathrm{PD}-\mathrm{L} 1^{+}$recurrence tumor cells may prevent T-cell activation from killing them via reducing CD80-CD28 interactions. If this speculation is true, it means that tumor immunotherapy plus chemotherapy or targeted therapy may be more effective than immune checkpoint blockade as a single agent for patients with recurrent HCC.

Overall, this study provided a comprehensive single-cell profiling in early-relapse HCC and explored the immunologic mechanisms of tumor relapse for the first time. It highlighted the potential significance of targeting immune contexture of HCC, and provided a solid foundation for the development of novel therapeutic strategies for recurrent HCC.

\section{Author's Contribution}

The author read and approved the final manuscript.

Funding

None.

Conflict of Interest

None declared.

\section{References}

1 Sung H, Ferlay J, Siegel RL, et al. Global cancer statistics 2020: GLOBOCAN estimates of incidence and mortality worldwide for 36 cancers in 185 countries. CA Cancer J Clin 2021;71(3):209-249

2 Cao M, Li H, Sun D, Chen W. Cancer burden of major cancers in China: a need for sustainable actions. Cancer Commun (Lond) 2020;40(05):205-210

3 Feng RM, Zong YN, Cao SM, Xu RH. Current cancer situation in China: good or bad news from the 2018 Global Cancer Statistics? Cancer Commun (Lond) 2019;39(01):22

4 Singal AG, Lampertico P, Nahon P. Epidemiology and surveillance for hepatocellular carcinoma: new trends. J Hepatol 2020;72(02): 250-261

5 Zheng J, Kuk D, Gönen M, et al. Actual 10-year survivors after resection of hepatocellular carcinoma. Ann Surg Oncol 2017;24 (05):1358-1366

6 Rossi JF, Céballos P, Lu ZY. Immune precision medicine for cancer: a novel insight based on the efficiency of immune effector cells. Cancer Commun (Lond) 2019;39(01):34

7 Wu T, Wu X, Wang HY, Chen L. Immune contexture defined by single cell technology for prognosis prediction and immunotherapy guidance in cancer. Cancer Commun (Lond) 2019;39(01):21

8 Zheng C, Zheng L, Yoo JK, et al. Landscape of infiltrating T cells in liver cancer revealed by single-cell sequencing. Cell 2017;169 (07):1342-1356.e16

9 Sun Y, Wu L, Zhong Y, et al. Single-cell landscape of the ecosystem in early-relapse hepatocellular carcinoma. Cell 2021;184(02): 404-421.e16

10 Ding X, He M, Chan AWH, et al. Genomic and epigenomic features of primary and recurrent hepatocellular carcinomas. Gastroenterology 2019;157(06):1630-1645.e6

11 Chiossone L, Dumas PY, Vienne M, Vivier E. Natural killer cells and other innate lymphoid cells in cancer. Nat Rev Immunol 2018;18 (11):671-688

12 Obar JJ, Jellison ER, Sheridan BS, et al. Pathogen-induced inflammatory environment controls effector and memory CD8+ T cell differentiation. J Immunol 2011;187(10):4967-4978

13 Amsen D, van Gisbergen KPJM, Hombrink P, van Lier RAW. Tissueresident memory $\mathrm{T}$ cells at the center of immunity to solid tumors. Nat Immunol 2018;19(06):538-546

14 Chen L. Co-inhibitory molecules of the B7-CD28 family in the control of T-cell immunity. Nat Rev Immunol 2004;4(05): 336-347

15 Butte MJ, Keir ME, Phamduy TB, Sharpe AH, Freeman GJ. Programmed death- 1 ligand 1 interacts specifically with the B7-1 costimulatory molecule to inhibit $\mathrm{T}$ cell responses. Immunity 2007;27(01):111-122 